\title{
Determination of Age using Traditional Techniques Such as Hand MRI, Face Recognition, Versus Building Neural Networks and Image Processing Techniques.
}

\author{
Dheeraj V. C., Karthik Holla A., Deeksha G., Nishchitha A., Kishore G.R.
}

\begin{abstract}
Technological advancement in forensic imaging have tremendous flow on benefits to the professional practice of forensic anthropology, not only in respect of case-work analyses, but also in facilitating empirical research that has validated and improved existing, and introduced novels, methods. The detail contribution include the spatial features of a dental $x$-ray image in a dental featured database. Determining the additional information it solves the model by Lagrange multiplier. Functional neural networks developed progressively with age. In dentistry, clinical radiographs reverberate the intensity loss of an $X$-ray when being transmitted through the mandibular objects. Dental examination is one of the most important parameter that help us to identify the age of an individual. Teeth are known to aid in personal identification as well as in addition to the age determination as they are highly durable to resist the effect of reaction by chemical and other environmental factors.
\end{abstract}

Keywords: Forensic science, Radiographic strategies, Dental age estimation, Data analysis and interpretation, Non-invasive methods.

\section{INTRODUCTION}

In the field of medical sciences, where age and gender can be determined using the bones and teeth as medium where in we examine the structure,patterns, and chronological appearances and so on. The people's age relay on a numerous factors including genetic inheritance, lifestyle and mental health. Distinguishing chronological age in both worms and humans is easy - count forward from birth. But

Revised Manuscript Received on February 05, 2020.

* Correspondence Author

Dheeraj V. C.*, Department of Information Science and Engineering, Jyothy Institute of Technology, Bengaluru, India.

Email: dheerajvc10@gmail.com

Karthik Holla A., Department of Information Science and Engineering,

Jyothy Institute of Technology, Bengaluru, India.

Email: karthikhollaa@gmail.com

Deeksha G., Department of Information Science and Engineering, Jyothy Institute of Technology, Bengaluru, India.

Email: deeksha199830@gmail.com

Nishchitha A., Department of Information Science and Engineering, Jyothy Institute of Technology, Bengaluru, India.

Email: nishchithaashok1012@gmail.com

Kishore G. R., Department of Information Science and Engineering, Jyothy Institute of Technology, Bengaluru, India. Email: kishoregr31@gmail.com

(C) The Authors. Published by Blue Eyes Intelligence Engineering and Sciences Publication (BEIESP). This is an open access article under the CC BY-NC-ND license (http://creativecommons.org/licenses/by-nc-nd/4.0/) distinguishing physiological age i.e. appearances of a person has remained subjective based on how someone looks or functions. In humans, sex is determined by five factors during birth time where in if there is presence or absence of a Y chromosome, the type of gonads, the sex hormones, the internal genitalia and the external genitalia.

In today's forensic technology determination of age and gender has got its vital importance in forensic sciences and medical sciences where in, that includes a variety of results such as, some disastrous events which makes difficult to estimate age and gender detection as such that it has got its importance for people. these includes technologies such as Machine Learning, Image processing,Artificial Neural networks etc has got its major role in detection of age and gender. In present day age estimation techniques, undamaged or unbroken or preserved bone, teeth, etc. are used for research purpose using dead human bodies. Especially in the domain of forensic medicine, age estimation process may be required for cases where there is a doubt about the actual age and hence no identity can be determined. In early days age of a species is found through rocks which were the decayed part of that species hence the archeological scientists would determine based on decay period and calculations on it. So that it would help in building a biological profile of that species. Age estimation techniques are useful in demographics of a population so that an average age of an individual could be determined and examined. Age could be also determined from telomere in humans where telomere is a nucleotide sequences that are repetative at the end of each chromosomes acts a chief factor in age estimation of a living individual through the length of a telomere. The length is main aspect of age determining factor in humans other than dental $x$-rays, as an living individual grows day by day his telomere length would decrease along with time. If an individual is suffering from a disease such as oncogenic growth in cells, it would affect the length of an telomere in living individual. The Age Estimation is an important activity that is frequently carried out in the medical legal practice, administration of justice often requires estimation of age of an individual in litigation involving both civil as well as criminal cases such as issues related to concerned employment and retirement where the age is required, a person can not be employed if his age below 18 years and usually retires at the age of 60 or 62 or 65 depending upon the governmental policies.

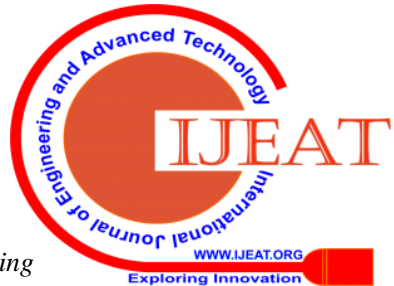


The marriages for example the age for the legal age of marriage of girls is 18 where as the legal age of marriage for boys is 21 years. So, even in the decomposed bodies or the badly charred bodies the teeth help us in approximately giving the age of the person and many other findings as well they may also be helpful when no other way of identification in age estimation is available such as in cases where only skeleton is available for examination so in that case the question regarding the bone is doesn't arise so again it is the teeth which becomes most important sometimes even a single tooth may help in establishing the identity or age of an individual.

\section{PROPOSED METHODOLOGY}

\section{A.Image processing}

[1] [4] The growth of age is usually determined by using texture of the skin, face appearance and Skin. The fate features Will change with the time, so we can determine or predict the actual age by considering wrinkles over the face in an image, by recognizing the wrinkle structure from the image and they are grouped by fuzzy c-mass clustering algorithm. The human features which are used for biometric authentication are unique and they are thumbprint, facial features, voice, iris and retina of the eye are widely used. But some of the features on face changes along with the age of human beings this is as depicted in the Fig. 1 and we need to update the database very often and doing this is very tedious .so this feature will help us to determine the person age.

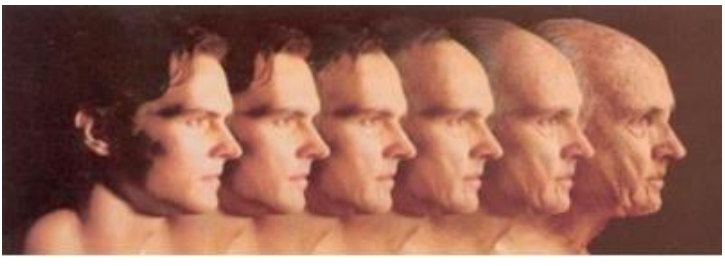

Fig. 1.progression of the human age.

The method that is implementing here has four different stages: Pre-processing, extraction of facial feature, categorization and estimation. We basically use two data sets the first one is training data set and the second is testing data set. In the first data set i.e. the training data set has age which is already known and in the second data set i.e. the test data set the age will be unknown which is needed to be found. With the help of MATLAB methods pre-processing of images is done, it is done by following steps, the initial process is getting the rectangular area of face in the image as depicted in the picture Fig. 2a. The next process is to detect pair of eyes, mouth, nose and chin as depicted in the picture Fig. 2b.
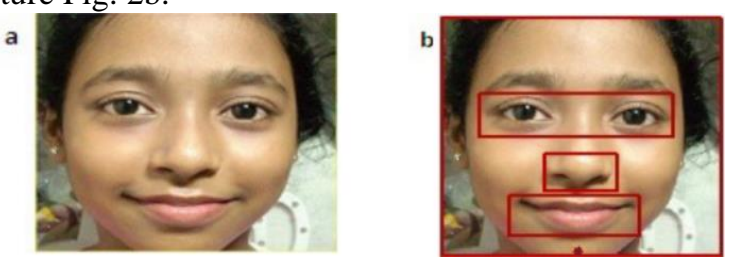

Fig. 2. (a) image of face identification. (b) image of region selection in the face.

The features in the wrinkle are calculated by using the portion in the forehead, checks upper portion, regions of the eyelids as depicted in the picture Fig. 3.After the location of the left eye $\left(\mathrm{x}_{\mathrm{l}}, \mathrm{y}_{\mathrm{l}}\right)$ and right eye $\left(\mathrm{x}_{\mathrm{r}}, \mathrm{y}_{\mathrm{r}}\right)$ mid position ,then the position between the eyeballs $\mathrm{d}$ is determined by the formula,

$$
d=\sqrt{ }(y r-y l)^{2}(x r-x l)^{2}
$$

a

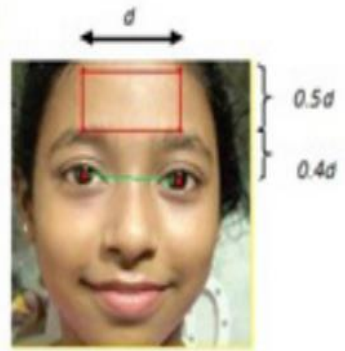

b

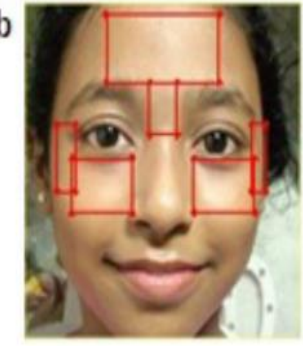

Fig. 3. (a) Forehead marking. (b)Marking the area of wrinkles over the image.

The next stage is feature extraction: The extraction of local and global features is done by images of face. The ratios of distance of forehead, nose, lips, right eyeball, chin, and left eyeball are the global feature. Wrinkles on face portions of forehead region, mid portion of eyebrows, corner regions of the eyes, eyelids are used as local feature. Using the distance of five parts and features that are extracted namely f1 to $\mathrm{f} 6$ are computed by the formula:

$\mathrm{f1}=$ (distance from right to left eye ball) / (distance from nose to eye)

$\mathrm{f} 2=($ distance from right to left eye ball) / (distance from lip to eye)

$\mathrm{f} 3=$ (distance from right to left eye ball) / (distance from chin to eye)

$\mathrm{f} 4=$ (distance from eye to nose) / (distance from lip to eye)

$\mathrm{f} 5=$ (distance from eye to nose) / (distance from chin to eye)

$\mathrm{f} 6=$ (distance from eye to chin) / (distance from chin to virtual top of head)

Initially the image of face is converted to image of gray scale. Later it allowed through technique called canny edge detection. In which the output is a binary image containing edges of the wrinkle as depicted in figure Fig. 4(a). In the facial image the wrinkle present are represented by the white pixels. The white pixel represent value 1 , and the black pixel represent value 0 in a binary image. So, wrinkle present on the face are directly proportional to summation of the pixel values of wrinkle area in binary face image, which is as shown in Fig. 4(b).
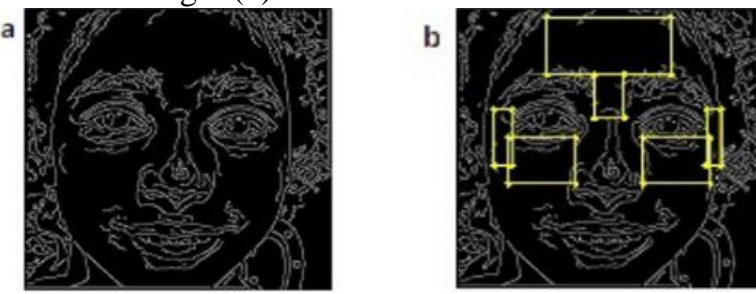

Fig. 4. (a) Image of face edge detection. (b) Image of boundaries of wrinkle area on face. 
F7= (summation of pixel values obtained in the forehead portion by summation of the pixels present in the forehead portion) + (summation of pixel values obtained by eyelid of the left eye by summation of pixels present in the eyelid of left eye) + (summation of pixel values obtained by eyelid of the right eye by summation of pixels present in the eyelid of right eye) + ( summation of pixel values obtained by corner of left eye by summation of pixels present in the corner of left eye) + (summation of pixel values obtained by corner of right eye by summation of pixels present in the corner of right eye).

The graph of age versus features that are obtained are individually plotted. $\mathrm{f7}$ will be used for classification of age.

$\mathrm{AGE}_{\mathrm{i}}=$ is the appropriate age of person with image I

$\mathrm{F}_{\mathrm{i}}=\mathrm{f} 7$ with image of face I

The value of $\mathrm{i}$ ranges from 1 to $\mathrm{m}$ for training images of face having count as $\mathrm{m}$.

Then we use classification, With the help of f7(wrinkle feature), the images of face will be classified with the help of algorithm called fuzzy C-Means clustering which would help us to get the membership value for all the cluster that have been formed. The membership value of $\mathrm{k}^{\text {th }}$ cluster having image $\mathrm{i}$ is given by $\mathrm{P}_{\mathrm{ik}}$.

The number of clusters as $n, \sum \mathrm{P}_{\mathrm{ik}}=1$ for all $\mathrm{i}$ where $\mathrm{k}$ varies from 1 to $\mathrm{n}$. For individual image I, we shall obtain the greatest membership value among the clusters. If $\mathrm{P}_{\mathrm{ik}}$ is the greatest value of $\left(\mathrm{P}_{11}, \mathrm{P}_{\mathrm{i} 2}, \mathrm{P}_{\mathrm{i} 3}, \ldots, \mathrm{P}_{\text {in }}\right)$ for image $\mathrm{i}$ belongs to cluster $\mathrm{k}$. So, every image having maximum membership value are clustered together.

And the final stage is age estimation, The mean age of individual cluster is obtained using data set used for training. Mean age of each clusters $k\left(A V G_{k}\right)$ is obtained by.

$$
A G_{k}=\left(\sum A G E_{k}\right) / N_{k}
$$

$\mathrm{AGE}_{\mathrm{k}}$ is representing the age of face image $\mathrm{i}$ used for training which is present in cluster $\mathrm{k}$ and $\mathrm{N}_{\mathrm{k}}$ is representing the count of images of faces used in training which belongs to the cluster $\mathrm{k}$. The estimation of age for face image which is given to test is obtained by applying the formula.

$$
\mathrm{E}-\mathrm{AGE}_{\mathrm{i}}=\Sigma\left(\mathrm{P}_{\mathrm{ik}} * \mathrm{AVG}_{\mathrm{k}}\right)
$$

There are many traditional methods for determining age in forensic science that includes Invasive methods are biomarkers, root dentine translucency, and incremental lines, There are four different categories for dental age estimation. Hence, this article overviews the different techniques involved in dental age estimation in adults. A preliminary estimation of age can be necessarily seen based on the presence of secondary sexual characteristics that become prominent when puberty is attained for example, breast development either this might be reduced breast in males and well developed breast as in females to feed their young ones, the development of pubic hair, and the laryngeal presence especially in males has its high priority. These characteristics develop as a result of sexual maturity. These features are the chief evidence for sexual difference between a group of population, that uses as a tool for evaluating age within a living communication that is obvious. There are many factors that can either inhibit the development of secondary sexual characteristics, most notable of which is varied facts in a similar group of population, geographic location, and individual body shape or size. A multidisciplinary evaluation that not only includes a physical and developmental evaluation but also a psychological assessment for the suspected juveniles legally.

Hence these are the traditional estimation for determining age wherein that is primarily concerned with DNA responsive factors for morphological,physiological,biological and psychological factors affecting age of a person by his appearances behaviour,mentality, maturity of conduct and so on hence now we move on to the new techniques involving technologies such as learning model, neural networks, machine learning algorithms, image processing techniques.

The primary objective of determining age and gender is by using neural network involving a multi layer perceptron model in image processing techniques.This involves collection, extraction, transformation, processing of x-ray images of teeth for age detection as a primary goal collectively in a teeth database. In human body the time taken to develop a tissue or an organ is optimum but whereas in the development of human teeth is a complex and sequential process that involves much more time than any other organ as a whole beginning with 6 weeks in utero and ending at 18-25 years of age. The dental development is likely to be affected with external factors such as environmental factors,chronological factors and nutritional factors along with time, which act as an chief indicator for dental mineralization and developments in sub adults compared to skeletal development that is more accurate. Accordingly, the primary factor for determining age is radiographic visualization and analysis of tooth formation stages are the foundation of many established age estimation techniques."The sequence of eruption and tooth count may be the only way to estimate the age of a living infant, as radiographs are contraindicated in this age group and the difficulty in obtaining a radiographs from an infant are predictable".There are many age estimation techniques that involve the above philosophy as a tool.

Hence numerous methodologies are involved in determination of age detection that would help in a serious and disasterous matters and also in unpredictable state of things in forensic science and medical technology to help it out in a efficient way using perceptron neural networks and image processing techniques.

\section{B. Multilayer Artificial neural network}

\section{1) The neuron}

Let us now consider a actual, real life neurons which are smeared on to the glass, colored a little bit and they are observed through microscope, so this is what they look like we can see quite an interesting structure, a body and a lot different tails kind of branches coming out of them. 
How can we recreate a neuron in a machine? : Because, we really need to recreate the neuron in a machine since the purpose of neural network is to mimic how the human brain works, by doing this we can create an amazing structure for machine to be able to learn, So our challenge right now is, our very first step is to create an Artificial neural network is to recreate a neuron.

Its got a body that's the main part of neuron and then its got some branches at the top which are called dendrites and its also got an axon which is that long tail of the neuron.

The key point to understand here is the neurons bt themselves are pretty much useless, its like an ant in which only one ant can't build an ant tower. Neuron also sometimes called node, the neuron gets some input signals and it has an output signals then this input signals we going to represent them with other neurons as well.

\section{2) Gradient Descent}

In order for a neural network to learn what needs to happen is Backpropagation and that is when the error, the difference or the sum of square differences between two layers is backpropagated through the neural network and the weights are adjusted accordingly. In order to implement this backpropagation technique we have used Brute force approach, when we take lots of different possible weights and look at them and see which one looks best.

We need to know whats happens in the neural network we know that there is a process called Forward propagation where the information is entered into the input layer and then is propagated forward to get our output values and then we compare those to the actual values that we have in our training set and then we calculate the errors. Then the errors are backpropagated through the network in the opposite direction and that allows us to train the neural network by adjusting the weights.

So, the key important thing to remember here is that backpropagation is an advanced algorithm driven by very interesting and sophisticated mathematics which allows us to adjust the weights, all of them at the same time all of the weights are adjusted simultaneously.

So, if we were doing this manually, or if we were coming up with a different type of algorithm then even if we calculated the error and then we were trying to understand what effect each of the weights has on the error, would have to somehow adjust each weights independently or individually. The huge advantage of backpropagation, and there is a key thing to remember is that during the process of backpropagation, simply because of the way the algorithm is structured, it is able to adjust all the weights at the same time so it basically know which part of the error each of your weights is the neural network is responsible for.

\section{3) Training the neural network}

Step 1. Randomly initiate the weights to small member close to 0(but not 0).

Step 2. Input the first observation of your dataset in the input layer each feature in one input node.

Step 3. Forward-Propagation from left to right the neuron are activated in a way that the impact of each neuron's activation is limited by the weights, propagate the activations until getting the predicted result $y$.

Step 4. Compare the predicted result to the actual result, measure the generated error.

Step 5. Backpropagation from right to left the error is backpropagated, update the weights according to hoe much they are responsible for the error. The learning rate decides by how much we update the weights.

Step 6. Repeat the steps 1 to 5 and update the weights after each observation (Reinforcement learning) or Repeat the steps 1 to 5 but update the weights only after a batch of observations (Batch learning).

\section{4) The Bias Variance tradeoff}

The Bias Variance tradeoff is that fact to train a model that will not only be accurate, but also should not have too much variance of accuracy, when we train it several times. And what happens for our ANN? : When the model is trained and have got two different accuracies and so now the model is not very sure which one of their two accuracies it should take, to evaluate the model performance and so, we need to optimise our way to evaluate our models. The best way is to split our dataset into training set and test set and trained our model on the training set and we tested its performance on the test set that the correct way of evaluating the model performance, but thats not the best one because we actual have the variance problem.

The Bias Variance can be explained by the fact that when we get accuracy on the test set, if we run the model again and test again its performance on another test set well we can get a very different accuracy.

So, judging our model performance only on one accuracy on one test set is actually not super relevant, there is a technique called K-Fold Cross Validation that improves this a lot.

\section{5) K-Fold Cross Validation}

K-Fold Cross Validation will fix the variance problem, and how will it fix it?

It will fix it by splitting the training set into 10 folds when $\mathrm{k}$ equals 10 , and most of the time, $\mathrm{k}$ is equal to 10 and we train our model on 9 folds and tested it on the last remaining fold, and since with 10 folds we can make 10 different combinations of 9 folds to train a model and 1 fold to test it. That means we can train the model and test the model on ten combinations of training and test set. And that will already give us much better idea of the model performance because, what we can do afterwards is to take the average of the different accuracies of the ten evaluations and also compute the standard derivation to have a look at the variance. So, eventually the analysis will be much more relevant and besides we will know which four categories will be, because if we get a good accuracy on a small variance then it is low bias low Variance. So, K-Fold Cross Validation is very useful and besides, our performance analysis is much more relevant.

\section{6) Improving the ANN}

Overfitting is when the model as trained too much on the training set, too much that it becomes much less performance on the test set and we can observe this when we have large difference of accuracies between training set and test set. 
Generally, when overfitting happens you have a much higher accuracy on the training set than the test set, and another way to detect overfitting is when you observe a high variance when applying $\mathrm{k}$-fold cross validation because indeed when its overfitted on the training set, that is when the model learn too much, well sometimes it will succeed on a new observations in one test set, when the correlations are similar to what the model learned too much and sometimes the model won't succeed on other test sets because the correlation that is learned too much, unfortunately don't apply to these test sets, and therefore in your vector of accuracies that are obtained with k-fold cross validations, you have some high accuracies and high variance and that's how you detect overfitting.

\section{7) Tuning the ANN}

Tuning the ANN is done through careful cross validation and the solution to succeed the best tool to use to accomplish the goal is parameter tuning. So what is parameter tuning?

We have two types of parameters, we have the parameters that are study from the model during the training and these are weights and we have some other parameters that stay fixed and there parameters are called hyper parameters. So, for example these hyper parameters are the number of a epoch, the batch size, the optimizer or the number of neurons in the layer and when we train our ANN well we trained it with some fixed values of these hyper parameters, but may be that bt taking some other values, we would get to better accuracy overall with careful cross validation.

It consists of finding the best values of these hyper parameters and do this with techniques called grid search that basically will test several combinations of these values and will eventually return the best selection, the best choice that leads to the best accuracy with careful cross validation.

\section{8) Tooth orientation}

Here we have used the technique of Artificial Neural Networks in order to use the machine learning techniques to inspire by the biological nervous system which are known as comuter systems.

The layers which are not present at the input layers are introduced in beteen the layers as the new layers to solve the non-linear problems. We use multiple neural networks to study about the problems which arise through the classification and regression.

$$
\mathrm{y}_{\mathrm{m}}=\mathrm{f}\left(\sum \mathrm{w}_{\mathrm{nm}} \mathrm{x}_{\mathrm{m}}\right)
$$

In MP, each input is indexed with $\mathrm{n}$ in the hidden layer, which collects all the weights by multiplying weights $\mathrm{w}$ and input $\mathrm{x}$ from the above equation(1). Where, $\mathrm{f}$ is the activation function and require to tranfer the input signal into neurons. $\mathrm{N}$ is the output value from the output layers of MP.


Fig5. Teeth orientation

\section{C.Age estimation using hand MRI}

The demand for bone age estimation of individuals and humans has recently been increased in permitted medical applications. The critical disadvantage of bone age estimation procedures based on $\mathrm{X}$-rays is a radiation exposure, because many in many places, they prohibit the scanning involving radiation of ionizing without diagnostic reason. Age estimation from radiological data plays major role in medical treatment. The methods for roughly calculated unrevealed age in kids and teenagers are on the basis of visual inspection of bone in hand MRI. The process of bone formation is most excellently followed in long bones and radiotherapist inspect hand bones caused by considerable amount of bones that are observable in MRI images, with reality the advancement of age is not concurrent for the bones of our hands. Over past few years, analysis in estimation of age has revealed interest in attractive MRI as dissipation free possibilities to evolve plans for bone formation advancement with respect to the bone evolution.

In age evaluation methods, all hand bones are differentiated at the same time to best coordinate image from the X-rays. The GP method is selected because it is not difficult to use and apply. Radiologists have to extract information from the bones in which the GP method is prone to intra- and intervariability. Single estimation are moved to bone formation that are combined with a previously defined result.

A serious disadvantage of estimating age technique is exposed to radiation. This is later amplified in medical applications, where several structures are examined for the extension of age estimation range beyond 18 years. For multiple age estimation, wisdom teeth is radiographed and computed tomography of bones are employed to hand radiographs, by significantly increasing the amount of ionized radiation.

Aging is treated as a global developmental process without the requirement for fusion schemes or pre-defined nonlinear functions.

\section{Algorithms}

Initially, Radigraphic denta x-ray images was created with teeth database. There are 1300 teeth images in the database. Every single teeth is having the details of age, number and count.

(AGE_TOOTHNUMBER_COUNT.png), for example, a 20 year old has a tooth number $19,2^{\text {nd }}$ tooth is recorded as follows 20_19_2.png

If the age of that human identifed by tooth if then recorded in the database.

All the images of the $\mathrm{X}$-rays are in equal size of $150 * 150$ with naming of all the images with A1,A2,A3,A4 methods are applied to the images which are created in the database respectively.

Algorithm-1: To Prepare Input to the Networks 


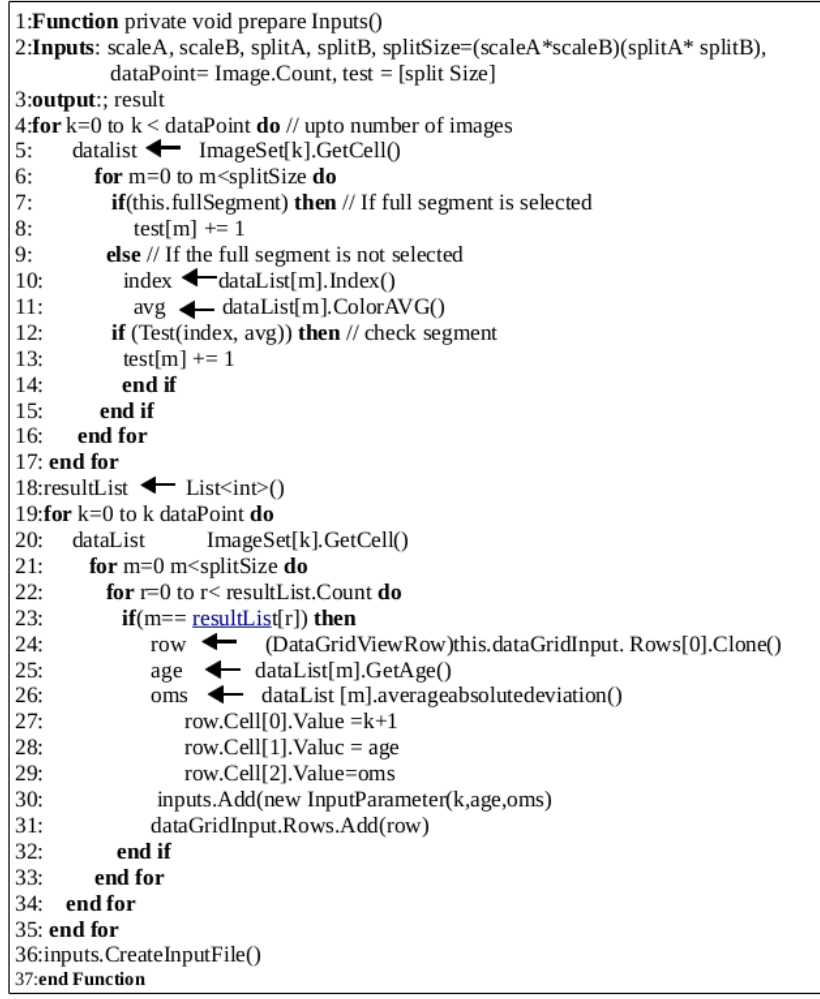

The above algorithm og to prepare inputs and send to the built networks which specifies with the preparation of inputs which are made as a images to the database to increase the performance of the neural network during the input taken to the network. The images are of white some are of black, they not represent the images to the network as input data.

Algorithm-2:Test function

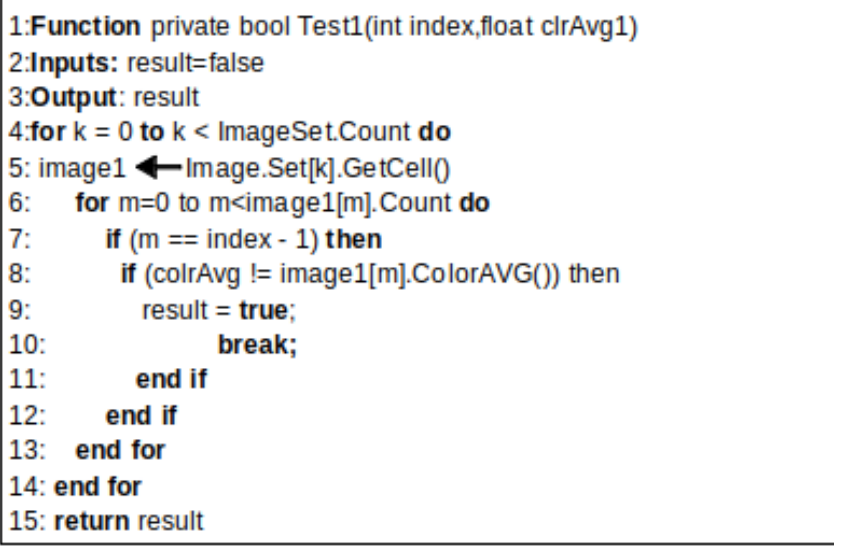

The test function is the $2^{\text {nd }}$ algorithm below written to be used as the function for the further operations. The algorithm for test function consists of nested function i.e. the function which is different from within another function.

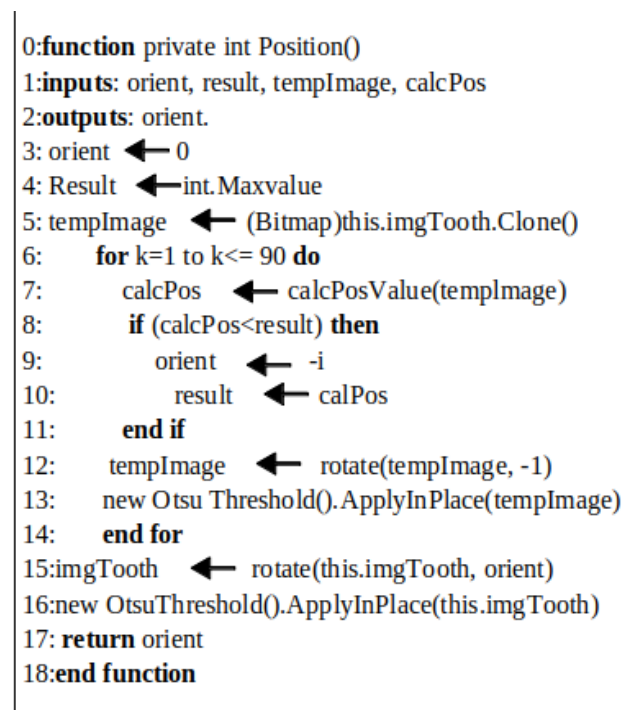

Algorithm 3 is an important algorithm which specifies the position and the orientation of the tooth into 90 degree step by step from $1-90$ degree cycles from $\mathrm{x}$ point indicated in the figure provided below,

At each angle step, the image calculates the position value using calcPos() function then the y values are summed at the image top of the tooth.

This algorithm verifies whether the tooth is in the accurate position perpendicular to the horizontal plane at $\mathrm{x}$ point.

\section{RESULTS}

The input parameter taken to the results as a network is the rate of learing with the values 0.1 and the sigmoid value is 2. Other input parameters are varying accordingly for each traning set of data taken as input to the Network.

When we refer to the table 1 , we infer that the input image taken to the network considering of the teeth between the ages of 4 and 15 years old are shown.

Table- I: Input images given to the network.

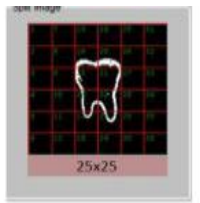

A1

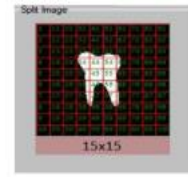

A4

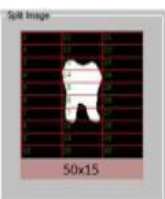

A2



A5

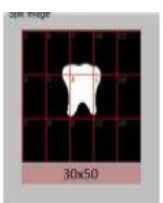

A3

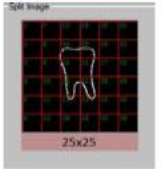

A6

Algorithm-3: Position 
Table- II: Input parameters infered from the $x$-ray image after segmentation

\begin{tabular}{|l|l|l|l|l|l|}
\hline Notation & \multicolumn{1}{|c|}{$\begin{array}{c}\text { Size } \\
\text { Count }\end{array}$} & $\begin{array}{c}\text { Input } \\
\text { Count }\end{array}$ & \multicolumn{1}{|c|}{ Epoch } & $\begin{array}{c}\text { Hidden } \\
\text { layer } \\
\text { count }\end{array}$ & Error \\
\hline A1 & $15 * 15$ & $100 * 250$ & 31876 & 22 & 0.1536 \\
\hline A1NFS & $15 * 15$ & $55 * 250$ & 31978 & 22 & 0.1546 \\
\hline A2 & $25 * 25$ & $57 * 233$ & 47834 & 22 & 0.1566 \\
\hline A2NFS & $25 * 25$ & $36 * 261$ & 47835 & 22 & 0.1566 \\
\hline A3 & $25 * 25$ & $23 * 256$ & 32225 & 22 & 0.3153 \\
\hline A3NFS & $25 * 25$ & $45 * 233$ & 124573 & 22 & 0.3187 \\
\hline A4 & $50 * 50$ & $15 * 260$ & 123475 & 22 & 0.3193 \\
\hline A4NFS & $50 * 50$ & $9 * 260$ & 16734 & 22 & 0.2434 \\
\hline A5 & $30 * 30$ & $13 * 245$ & 24159 & 22 & 0.2893 \\
\hline A5NFS & $30 * 30$ & $17 * 234$ & 24159 & 22 & 0.4600 \\
\hline
\end{tabular}

NFS: NotFullSegment

Table 2 in the above mention the error values in the network earlier produced before calculating the age in the following table.

Table- III: Network Results

\begin{tabular}{|l|l|l|l|l|}
\hline Notation & \multicolumn{1}{|c|}{ Real age } & $\begin{array}{c}\text { Estimated } \\
\text { age }\end{array}$ & Difference & $\begin{array}{c}\text { Classification } \\
\text { success }\end{array}$ \\
\hline A1 & 5.5 & 4.5 & \pm 1 & $\% 99.99999$ \\
\hline A1NFS & 5.5 & 4.5 & \pm 1 & $\% 99.99322$ \\
\hline A1 & 6 & 4 & \pm 2 & $\% 99.99999$ \\
\hline A1NFS & 6 & 6 & \pm 0 & $\% 99.99999$ \\
\hline
\end{tabular}

\begin{tabular}{|l|l|l|l|l|}
\hline A1 & 8.5 & 9.5 & \pm 1 & $\% 99.99999$ \\
\hline A2 & 8.5 & 9 & \pm .5 & $\% 99.99999$ \\
\hline A2NFS & 6.5 & 9 & \pm 2.5 & $\% 99.99088$ \\
\hline A2 & 6.5 & 4.5 & \pm 2 & $\% 99.99999$ \\
\hline A2NFS & 6.5 & 5.5 & \pm 1 & $\% 99.99341$ \\
\hline A2 & 6.5 & 4.5 & \pm 2 & $\% 99.99999$ \\
\hline A2NFS & 9 & 4 & \pm 5 & $\% 99.98674$ \\
\hline A3 & 9 & 4 & \pm 5 & $\% 99.98765$ \\
\hline A3NFS & 8.5 & 6.5 & \pm 2 & $\% 99.99999$ \\
\hline A3 & 6.5 & 7 & \pm 0.5 & $\% 99.99999$ \\
\hline A4 & 7.5 & 9.5 & \pm 2 & $\% 99.99999$ \\
\hline A4 & 9 & 7 & \pm 2 & $\% 99.99999$ \\
\hline A4NFS & 9 & 4.5 & \pm 4.5 & $\% 99.96554$ \\
\hline A4 & 5.5 & 9.5 & \pm 4 & $\% 99.99543$ \\
\hline A5 & 6 & 8 & \pm 2 & $\% 99.99999$ \\
\hline A5NFS & 6 & 6.5 & \pm 0.5 & $\% 99.99999$ \\
\hline A5 & 8 & 8 & \pm 0 & $\% 99.99999$ \\
\hline A5NFS & 8 & 8 & \pm 0 & $\% 99.99999$ \\
\hline A6 & 9 & 9.5 & \pm 0.5 & $\% 99.99999$ \\
\hline A6NFS & 9 & 8 & \pm 1 & $\% 99.99999$ \\
\hline
\end{tabular}

The result and the classification is shown on table 3 using trained and classification ratio. The estimated age was produced which was determined between 4 and 15 years old and error rate was much lesser comparing with the previous trained examples.

\section{GAP IDENTIFICATION}

TABLE I. Comparative Study

\begin{tabular}{|c|c|c|c|c|c|}
\hline $\begin{array}{l}\text { SL } \\
\text { NO }\end{array}$ & Authors(s) & Year & Approach & Description & Gap Identification \\
\hline 1 & $\begin{array}{l}\text { Ranjan Janaa, } \\
\text { Debaleena } \\
\text { Dattaa, } \\
\text { Rituparna } \\
\text { Sahaa }\end{array}$ & 2014 & $\begin{array}{l}\text { Using wrinkles } \\
\text { on the face }\end{array}$ & $\begin{array}{l}\text { Wrinkle areas on face are detected } \\
\text { and the wrinkle feature extraction is } \\
\text { done by using face image. Based on } \\
\text { the result of wrinkle features of } \\
\text { individual face image which will be } \\
\text { clustered with the help of the } \\
\text { algorithm fuzzy c-means clustering } \\
\text {.Later, age to be estimated is } \\
\text { obtained using the membership value } \\
\text { of each clusters and the mean age of } \\
\text { the respective cluster. }\end{array}$ & $\begin{array}{l}\text { Age estimation based on the } \\
\text { wrinkles on the face is not a good } \\
\text { solution, because the wrinkles on } \\
\text { the face can be a genetical } \\
\text { disorder and also the pollution } \\
\text { will affect the skin. }\end{array}$ \\
\hline 2 & $\begin{array}{l}\text { Darko Stern, } \\
\text { Christian } \\
\text { Payer, } \\
\text { Martin } \\
\text { Urschler }\end{array}$ & 2019 & Hand MRI & $\begin{array}{l}\text { The age is estimated using the MRI } \\
\text { scans of hand, the age is } \\
\text { comprehensively evaluate by using } \\
\text { the }\end{array}$ & $\begin{array}{l}\text { MRI is expensive and also this } \\
\text { may cause the skin infections for } \\
\text { sensitive people. And also the } \\
\text { growth of the bone is effected by } \\
\text { the food habits. }\end{array}$ \\
\hline
\end{tabular}


Determination of Age using Traditional Techniques Such as Hand MRI, Face Recognition, Versus Building Neural Networks and Image Processing Techniques.

\begin{tabular}{|c|c|c|c|c|c|}
\hline 3 & $\begin{array}{l}\text { Jana naue, } \\
\text { Hubb C.J. } \\
\text { Hoefsloot, }\end{array}$ & 2017 & $\begin{array}{l}\text { DNA } \\
\text { Methylation }\end{array}$ & $\begin{array}{l}\text { The age estimation technique can be } \\
\text { determined by dna methylation which } \\
\text { is adding methyl molecule to dna } \\
\text { hence inferring changes in the dna, } \\
\text { hence predicting forensic age. }\end{array}$ & $\begin{array}{l}\text { DNA methylation might be } \\
\text { efficient method but it cannot } \\
\text { differentiate the persons age } \\
\text { living capacity wherin a person } \\
\text { having bad habbits that affect his } \\
\text { physical environment. }\end{array}$ \\
\hline 4 & $\begin{array}{l}\text { Nameirakpam } \\
\text { dhanachandra, } \\
\text { Khumanthem } \\
\text { manglem }\end{array}$ & 2015 & $\begin{array}{l}\text { Image } \\
\text { segmentation } \\
\text { using } \mathrm{k} \text { means } \\
\text { clustering } \\
\end{array}$ & $\begin{array}{l}\text { Image segmentation is nothing but } \\
\text { classification of image into different } \\
\text { groups using } \mathrm{k} \text { means clustering } \\
\text { algorithm. }\end{array}$ & $\begin{array}{l}\text { To determine using image would } \\
\text { be difficulty if the image is not } \\
\text { proper. }\end{array}$ \\
\hline 5 & $\begin{array}{l}\text { Emre avuclu, } \\
\text { faith basciftci }\end{array}$ & 2018 & $\begin{array}{l}\text { Determination } \\
\text { of age and } \\
\text { gender usimg } \\
\text { neural networks }\end{array}$ & $\begin{array}{l}\text { Determination of age and gender } \\
\text { using neural networks in image } \\
\text { processing techniques }\end{array}$ & $\begin{array}{l}\text { Building and understanding } \\
\text { neural networks is difficulty. }\end{array}$ \\
\hline
\end{tabular}

\section{CONCLUSION}

A solution for estimation of age from $\mathrm{x}$-rays of hands are presented. The approach of this is not depending on the requirement for ionizing, that is severe in medical applications involving healthy persons. The evaluation of dissimilar ML methods disclosed in DCNN established few proceeds towards achieving a state of accuracy contrast with other x-ray based methods. The MRI dataset of few images,show that the explanation of prior knowledge on the anatomical information that is age relevant, is located is beneficial for DCNN to learn high calculated nonlinear backsliding problem.

It is assumed that in the presence of a bigger training models, need for such preprocessing method can be overcome.

The set of Database which was created earlier to determine age. The database initially consists of $1300 \mathrm{x}$-ray images taken online. The database include the segmented images of x-rays and also sketched of the boundaries of the teeth and grouping of images according to the age as shown in Fig. 5.

Hence I conclude that there are numerous methods where age can be estimated such as MRI Scan of hand, Neural Networks, Machine Learning Algorithms in a software approach, ,Artificial Neural Networks, Image processing using face,teeth bones etc,which is helpful in the field of medical and Sciences.

\section{ACKNOWLEDGMENT}

First and foremost, I wish to record my sincere gratitude to Management of Jyothy Institute of Technology and to my beloved Principal, Dr. Gopalakrishna, JIT, Bengaluru for his constant support and encouragement .

My sincere thanks to Dr. Harshvardan Tiwari, HOD, ISE, for his valuable suggestions for writing the paper.

\section{REFERENCES}

1. https://www.sciencedirect.com/science/article/pii/S187705091500190 $\underline{8}$ for face identification.

2. https://www.sciencedirect.com/science/article/pii/S136184151830479 1 for hand mri.

3. $\quad$ https://www.sciencedirect.com/science/article/pii/S187249731730164 3 for Chronological age prediction based on DNA methylation: Massive parallel sequencing and random forest regression.

4. https://www.sciencedirect.com/science/article/pii/S187705091501414 3

5. for Image Segmentation Using K -means Clustering Algorithm and Subtractive Clustering Algorithm.

6. https://www.researchgate.net/publication/283845399 Forensic age e stimation_in_living_individuals methodological_consideration_in th e_context_of_medico-legal_practice

7. for Forensic age estimation in living individuals: methodological considerations in the context of medico legal practice. HTTPS://WWW.SCIENCEDIRECT.COM/SCIENCE/ARTICLE/PII/S156849461 8303089. NEW APPROACHES TO DETERMINE AGE AND GENDER IN IMAGE PROCESSING TECHNIQUES USING MULTILAYER PERCEPTRON NEURAL NETWORK

8. Yılmazer Ö. Adli Tıp Kurumu"nda yaş tayininde kullanılan yöntemin verimlilik aç ısından değerlendirilmesi. Uzmanlık Tezi, İstanbul, 2006.

\section{AUTHORS PROFILE}

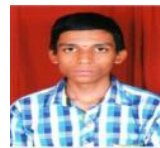

Dheeraj vc, is pursuing Bachelor's degree in nformation Science and Engineering at Jyothy Institute of technology, Bengaluru. Affiliated to VTU, Belagavi,He s certified in Data Mining course conducted by NPTEL, hand his areas of interest are C, Java, Python programming CSI. Email-id: anguages and Machine Learning. He is a member of

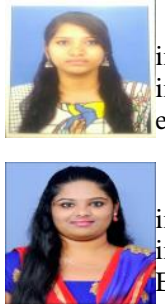

Nishchitha A, Pursuing my Bachelor's degree in information science and engineering branch at jyothy institute of technology .

email-id:nishchithaashok1012@gmail.com

Deeksha G, Pursuing my Bachelor's degree in information science and engineering branch at jyothy nstitute of technology

Email-id:deeksha199830@gmail.com
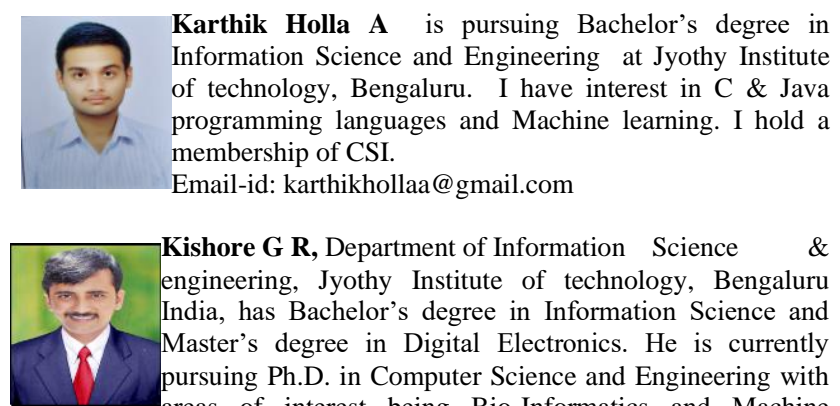

Kishore G R, Department of Information Science \& engineering, Jyothy Institute of technology, Bengaluru India, has Bachelor's degree in Information Science and Master's degree in Digital Electronics. He is currently pursuing Ph.D. in Computer Science and Engineering with areas of interest being Bio-Informatics and Machine learning. He is a life member of ISTE and CSI.

E-mail: kishoregr31@gmail.com 